\title{
Interactive comment on "Contributions of nitrated aromatic compounds to the light absorption of water-soluble and particulate brown carbon in different atmospheric environments in Germany and China" by Monique Teich et al.
}

Anonymous Referee \#2

Received and published: 28 September 2016

Summary and Overall Recommendation:

This study examines the contribution of nitrated aromatic compounds (NACs) to light absorption of aqueous particle extracts and particulate brown carbon $(\mathrm{BrC})$ from samples collected mainly from Germany and some from China. Aerosol samples were collected onto quartz fiber filters using high-volume filter samplers. The authors focus on 8 NACs, which included nitrophenols and nitrated salicylic acids), previously recognized from prior lab and field studies to contribute to BrC. The novelty in this study lies in the fact that the authors compare the contributions of NACs to WSOC mass and 
aqueous aerosol extract light absorption from 4 locations in Germany and 2 locations China. Mainly summer versus winter comparisons are made in Germany and only summer data are obtained from China. The spatial comparisons are quite interesting as the authors know when some of these sites are directly affected by biomass burning (BB events). Overall, this study will be of interest to many readers of ACP; however, there are a lot of technical issues that need to be addressed (as outlined below) before publication can be fully considered. In addition, it would have been more interesting if the authors would have gone further in the chemical characterization of the $\mathrm{BrC}$ components collected from these different locations and seasons, especially considering that the NACs didn't explain a larger fraction of the BrC mass. As the authors indicate in the last lines of their paper within the conclusions section, the exciting new results lie in identifying new tracers for $\mathrm{BrC}$ that indicate source and chemical process. With that said, I do think many researchers working in $\mathrm{BrC}$ aerosol will find this paper interesting due to the use of known BrC constituents (NACs) and comparing their trends between locations and seasons to gain insights into their potential sources. The comparison of the German winter sites to the Chinese summer sites isn't surprising, but it is compelling to see that BB likely contributed to the NACs concentrations at the German winter sites where as other sources (one I mention below in the specific comments section) contribute to NACs levels in China during summer.

Specific Comments:

1.) Important details missing for the high-volume filter sampling protocols:

How were the quartz fiber filters treated before sampling? Were they pre-combusted before sampling, and if so, at what temperatures and for how long? How were these filters stored after collection? Were they stored in pre-combusted Al foil packets or some other kind of container? How long were filters stored before chemical analyses and how did this affect the data presented here? Did the authors determine the recovery efficiencies of NACs from this filter media and was this considered into the calculations for their mass concentrations? The authors stated that the samples were stored at -20

Printer-friendly version

Discussion paper
Interactive

comment

C2 
C. Please clarify that this was under dark conditions too of course. Even though it may seem trivial, these details really should be added to the experimental section.

\section{2.) Extraction solvent:}

Can the authors comment on how well water extractions remove $\mathrm{BrC}$ constituents from the filters? Why wasn't another solvent, such as an organic solvent, considered as well in this study? I ask this question since HULIS-like species, which are likely oligomeric in nature, may not have been well removed from the filter media. As the authors know, HULIS-like species can contribute to the BrC fraction. Lin et al. (2014, ES\&T) found that the $\mathrm{BrC}$ fraction within IEPOX-derived SOA was highly oligomeric in nature but also less water soluble, so extracting the filters with an organic solvent was really important in discovering these light-absorbing oligomers. This study isn't the only one to consider this issue, but certainly a recent example to consider in terms of extraction solvent.

\section{3.) Levoglucosan:}

Since levoglucosan was quantified using IC coupled to PAD, how confident are the authors that there are no co-eluting species? I ask this question since GC/MS with prior derivatization tends to take this concern away due to its high chromatographic resolution.

4.) Changing the $\mathrm{pH}$ of aqueous extracts:

By intentionally making extracts acidic or basic, do the authors fear changes in the chemical composition could occur due to unwanted reactions? This is important to think about, especially if one is concerned about the presence of oligomeric species that could degrade via dehydration reactions or other types of unforeseen reactions. I think the authors need to comment on this potential issue. As an example, how might this affect the UV-Vis measurements? I can see this step you have introduced here being confusing to some of the readership of ACP.

5.) BB not the possible source of NACs at the Chinese sites: 
Were these NACs during summer in China associated with the photochemical oxidation of anthrpogenic VOCs, such as aromatics? Previous work, such as by the EPA group (Jaoui et al., studies) and Sato et al. (JPCA, 2008), have shown that the photochemical oxidation of aromatic VOCs in the presence of NOx yields NACs. If you collect filters from these experiments, they are brown. So it would be interesting to know if this is correlated with photochemical processing of VOCs (like aromatics) associated with traffic emissions.

6.) Page 10, Line 26:

The authors state "nighttime concentrations were found to be slightly higher than during the day." For statements like this one and elsewhere in the manuscript, is this statistically significant?

7.) Page 11, Line 11:

The authors state "The contribution of NACs to Abs(370 nm) was low for the campaigns Waldstein (summer) and Melpitz (summer)."

Probably not unexpected, right, especially since there is no BB influence or traffic influence? But are there other types of BrC constituents missing, such as those observed from monoterpenes in lab studies by the Laskin and Nizorodov groups? It would be interesting to know what is contributing to the small BrC levels..

Minor Comments:

1.) Fix the numbering of subsections in Section 2.

Interactive comment on Atmos. Chem. Phys. Discuss., doi:10.5194/acp-2016-647, 2016. 